\title{
The Impact of FcyRI Binding on Immuno-PET
}

\author{
Delphine Vivier*1, Sai Kiran Sharma*2, Pierre Adumeau ${ }^{1}$, Cindy Rodriguez ${ }^{1}$, Kimberly Fung ${ }^{1,3}$, and Brian M. Zeglis ${ }^{1-3}$ \\ ${ }^{1}$ Department of Chemistry, Hunter College of the City University of New York, New York, New York; ${ }^{2}$ Department of Radiology, \\ Memorial Sloan Kettering Cancer Center, New York, New York; and ${ }^{3}$ Ph.D. Program in Chemistry, The Graduate Center of the City \\ University of New York, New York, New York
}

\begin{abstract}
Antibodies are promising vectors for PET imaging. However, the high uptake of radioimmunoconjugates in nontarget tissues such as the liver and spleen hampers their performance as radiotracers. This off-target uptake can lead to suboptimal tumor-to-background activity concentration ratios, decreasing the contrast of images and reducing their diagnostic utility. A possible cause of this uptake is the sequestration of radioimmunoconjugates by immune cells bearing Fc-y-receptors (FcyR) that bind to the Fc regions of antibodies. Methods: Since the heavy chain glycans influence the affinity of FcyR for the Fc domain, we set out to investigate whether radioimmunoconjugates with truncated glycans would exhibit altered binding to FcyRl and, in turn, improved in vivo performance. Using the HER2-targeting antibody trastuzumab, we synthesized a series of desferrioxamine-bearing immunoconjugates with differing glycosylation states and interrogated their FcyRI binding via surface plasmon resonance, enzyme-linked immunosorbent assay, and flow cytometry. Furthermore, we labeled these immunoconjugates with ${ }^{89} \mathrm{Zr}$ and explored their biodistribution in athymic nude, NSG, and humanized NSG mice bearing human epidermal growth factor receptor 2-expressing human breast cancer xenografts. Results: We observed a strong correlation between the impaired in vitro FcyRI binding of deglycosylated immunoconjugates and significant decreases in the in vivo off-target uptake of the corresponding ${ }^{89} \mathrm{Zr}$ labeled radioimmunoconjugates (i.e., liver activity concentrations are reduced by $\sim 3.5$-fold in humanized NSG mice). These reductions in off-target uptake were accompanied by concomitant increases in the tumoral activity concentrations of the glycoengineered radioimmunoconjugates, ultimately yielding improved tumor-to-healthy organ contrast and higher quality PET images. Conclusion: Our findings suggest that the deglycosylation of antibodies represents a facile strategy for improving the quality of immuno-PET in animal models as well as in certain patient populations.
\end{abstract}

Key Words: glycans; Fc region; Fc receptor; FcyRI; PET; radioimmunoconjugate

J Nucl Med 2019; 60:1174-1182

DOI: 10.2967/jnumed.118.223636

$\mathbf{O}$

ver the past 2 decades, antibody-based PET (immuno-PET) has emerged as a clinically relevant technology for the staging, treatment planning, and treatment monitoring of cancer (1). Not

Received Dec. 20, 2018; revision accepted Jan. 1, 2018.

For correspondence or reprints contact: Brian M. Zeglis. 413 East 69th St., Hunter College of the City University of New York, New York, NY 10021.

E-mail: bz102@hunter.cuny.edu

${ }^{*}$ Contributed equally to this work.

Published online Feb. 7, 2019.

COPYRIGHT (C 2019 by the Society of Nuclear Medicine and Molecular Imaging. surprisingly, however, immuno-PET has its drawbacks. For example, the sluggish in vivo pharmacokinetics of antibodies means that sufficient image contrast can only be obtained more than $72 \mathrm{~h}$ after the administration of the tracer. Furthermore, the high molecular weight $(\sim 150 \mathrm{kDa})$ of full-length antibodies directs their clearance to the hepatobiliary system, often resulting in the accumulation of radioactivity in the healthy liver and spleen. A less well-described phenomenon that contributes to the nonspecific uptake of antibodies is their inherent ability to interact with the immune system. While the Fab portion of an antibody is responsible for binding to antigens, the $\mathrm{Fc}$ region engages in interactions with $\mathrm{Fc}$ receptors, most notably $\mathrm{Fc}-\boldsymbol{\gamma}$-receptors $(\mathrm{Fc} \gamma \mathrm{R})$ and the neonatal $\mathrm{Fc}$ receptor $(\mathrm{FcRn})$ (Fig. 1A). The binding of the latter facilitates the antibody's escape from lysosomal degradation, which in turn leads to its in vivo recycling and extended serum half-life. In contrast, the interactions between Fc $\gamma \mathrm{R}$ expressed on immune effector cells and the $\mathrm{Fc}$ region of antibodies can trigger antibodymediated therapeutic responses (2,3). Critically, the affinity of Fc $\gamma \mathrm{R}$ is sensitive to the glycosylation state of the antibody, whereas that of FcRn is not (4). Although interactions with FcyR are critical for therapeutic antibodies, they may not be favorable in the context of molecular imaging (5). Indeed, the immune response sparked by the binding of antibodies to $\mathrm{Fc} \gamma \mathrm{R}$ may lead to the sequestration of radioimmunoconjugates in healthy nontarget tissues, decreasing tumor-to-background contrast and dampening the quality and diagnostic utility of the images.

Several approaches to circumventing this issue have been proposed. For example, a great deal of attention has been dedicated to antibody fragments such as Fab and $\mathrm{F}(\mathrm{ab})_{2}$, which not only have more rapid pharmacokinetic profiles than full-length antibodies but also lack the Fc region responsible for interactions with the immune system. However, these traits undoubtedly come at a price: very high activity concentrations in the kidneys and tumoral activity concentrations that are only a fraction of those obtained using intact IgGs (6). Another strategy is predicated on genetically engineering the $\mathrm{Fc}$ region of an $\mathrm{IgG}$ to abrogate its binding with Fc $\gamma$ Rs on immune cells while maintaining its ability to bind FcRn (7). This method is effective, yet it is complex and expensive and requires specialized genetic engineering.

A more facile and modular approach may lie in manipulating the glycans of the $\mathrm{Fc}$ region. In recent years, the role of the $\mathrm{Fc}$ region in the in vivo behavior of antibodies has been the subject of increasing attention, and the role of glycosylation in the binding of IgG1 to Fc $\gamma \mathrm{R}$ has become a focal point (8). Indeed, IgGs contain a conserved $N$-linked glycosylation site-Asn-X-Ser/Thr, where $\mathrm{X}$ can be any amino acid except proline-within the $\mathrm{C}_{\mathrm{H}} 2$ domain of each heavy chain of the Fc region $(9,10)$. The $N$-linked complex glycan attached to each Asn297 is a biantennary heptasaccharide chain composed of $\mathrm{N}$-acetylglucosamine and mannose residues 


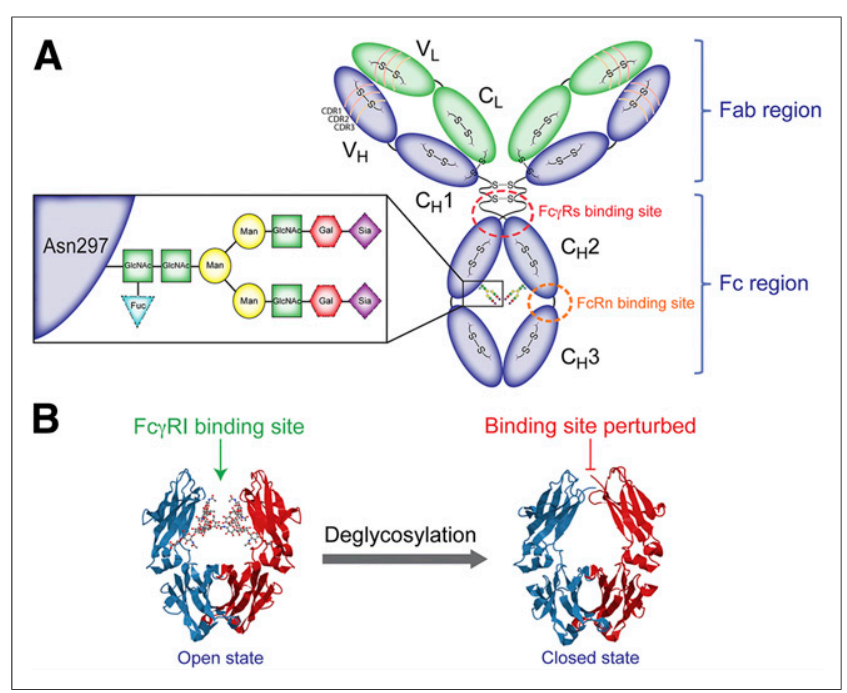

FIGURE 1. Antibody structure and FcyRI binding. (A) Detailed structure of an antibody with a magnified view of the glycans. (B) Cartoon depicting the influence of deglycosylation on the structure of the $\mathrm{Fc}$ region of an antibody and its binding to FcyRI.

that can be decorated with fucose, galactose, and sialic acid (Fig. 1A). Although the glycans are not directly involved in the antibody's interaction with $\mathrm{Fc} \gamma \mathrm{R}$, several studies have shown that these sugar chains control the 3-dimensional configuration of the $\mathrm{Fc}$ region and, as a result, the ability of $\mathrm{Fc}$ receptors to access the Fc-binding site (Fig. 1B) $(5,11,12)$. The deglycosylation of the $\mathrm{C}_{\mathrm{H}} 2$ domain of an IgG1 transforms the horseshoelike configuration of the native antibody to a closed state, blocking access to the Fc $\gamma \mathrm{R}$ binding site $(11,12)$. As a result, deglycosylated antibodies show reduced binding affinity for Fc $\gamma \mathrm{R}$. Critically, Fc $\gamma \mathrm{RI}$ is the only member of the Fc $\gamma \mathrm{R}$ family that has high affinity for the $\mathrm{Fc}$ region and is able to bind monomeric IgGs. The other Fc $\gamma \mathrm{R}-$ Fc $\gamma$ RII and Fc $\gamma$ RIII-have lower affinities for monomeric IgGs and prefer to bind to immune-complexes (13). In humans, Fc $\gamma \mathrm{RI}$ is expressed on the surface of monocytes, macrophages, and tissue-resident macrophages, such as Kupffer cells in the liver $(5,14)$. The deglycosylation of antibodies has previously been shown to improve the performance of near-infrared fluorescence imaging agents based on murine antibodies (15).

The core hypothesis of this investigation is that the deglycosylation of antibodies could produce "immune-silent" radioimmunoconjugates with improved in vivo performance. To this end, we produced several desferrioxamine (DFO)-bearing immunoconjugates of the human epidermal growth factor receptor 2 (HER2)targeting antibody trastuzumab with differing glycosylation states. Subsequently, we explored the influence of our glycoengineering efforts on the binding of these immunoconjugates with Fc $\gamma$ RI via surface plasmon resonance (SPR), enzyme-linked immunosorbent assay (ELISA), and flow cytometry. Finally, we labeled the DFObearing immunoconjugates with ${ }^{89} \mathrm{Zr}$ (half-life, $\sim 78.4 \mathrm{~h} ; 22.7 \%$ $\beta^{+} ; \mathrm{E}_{\beta+}=395.5 \mathrm{keV}$ ) and explored their in vivo behavior in 3 mouse models of HER2-expressing human breast cancer. A compelling correlation between the in vitro and in vivo results was observed: the ${ }^{89} \mathrm{Zr}$-DFO-trastuzumab radioimmunoconjugates that displayed abrogated binding to Fc $\gamma \mathrm{RI}$ in vitro exhibited improved performance in vivo, specifically decreased uptake in the healthy liver and spleen and increased retention in tumor tissue.

\section{MATERIALS AND METHODS}

All chemicals, unless otherwise noted, were acquired from Fisher Scientific and used as received without further purification. All water used was ultra-pure $\left(>18.2 \mathrm{M} \Omega \mathrm{cm}^{-1}\right)$, and dimethylsulfoxide was of molecular biology grade $(>99.9 \%)$. ${ }^{89} \mathrm{Zr}$ was produced and purified at Memorial Sloan Kettering Cancer Center (MSKCC) as reported elsewhere (16). Activity measurements were made using a CRC-15R Dose Calibrator (Capintec), and experimental samples were counted on an Automatic Wizard $^{3} \gamma$-counter (Perkin-Elmer). The synthesis, purification, electrophoresis, matrix-assisted laser desorption/ionization-time of flight (MALDI-TOF) mass spectrometry, and lectin culinaris agglutinin (LCA) blot analysis of DFO- ${ }^{\text {nss }}$ trastuzumab, DFO- ${ }^{\text {nss }}$ trastuzumab-PNGaseF, DFO- ${ }^{\text {nsstrastuzumab- }}$ EndoS, and DFO-sstrastuzumab-EndoS were performed according to previously published methods (17-20). Likewise, the radiolabeling and stability analysis of the ${ }^{89} \mathrm{Zr}$-labeled radioimmunoconjugates were performed according to established protocols (21). Cell culture and flow cytometry experiments with BT474, U937, and RAW264.7 cells were performed using a BD LSR-II instrument (BD Biosciences) using standard procedures (22). Finally, all in vivo experiments-including the establishment of BT474 xenografts as well as PET imaging and biodistribution studies - were performed according to published protocols approved by the Institutional Animal Care and Use Committees of MSKCC and Hunter College (22).

\section{In Vitro FcyRI Binding ELISA}

Recombinant mouse or human FcyRI/CD64, CF (R\&D Systems \#2074-FC-050 or \# 257-FC-050) was diluted to $10 \mu \mathrm{g} / \mathrm{mL}$ in sterile phosphate-buffered saline, and $100 \mu \mathrm{L} /$ well were coated overnight at $4^{\circ} \mathrm{C}$ onto an ELISA plate (Plates-Nunc MaxiSorp flat-bottom 96-well plate; Fisher Scientific) (23). After a brief blocking period (40 min with phosphate-buffered saline containing $10 \%$ fetal calf serum), the immunoconjugates were diluted in blocking buffer $(0.5$ or $50 \mu \mathrm{g} / \mathrm{mL})$, and $100 \mu \mathrm{L} /$ well were applied for $2 \mathrm{~h}$ at room temperature. So as not to disrupt the Fc-FcyRI interactions, the immunoconjugates were detected using 1:5,000 horseradish peroxidase-labeled anti-human IgG (JacksonImmunoResearch Laboratories). After a final wash step, 3,3',5,5'-tetramethylbenzidine (TMB) substrate was used to develop the horseradish peroxidase secondary antibody, and the color reaction was stopped with $2 \mathrm{~N} \mathrm{H}_{2} \mathrm{SO}_{4}$. Optical densities at 450 $\mathrm{nm}$ were determined using a SpectraMax i3 plate reader (Molecular Devices). Binding data were collected in triplicate, averaged, and plotted.

\section{SPR}

The kinetic constants $\left(\mathrm{k}_{\mathrm{a}}\right.$ and $\left.\mathrm{k}_{\mathrm{d}}\right)$ and binding affinity $\left(\mathrm{K}_{\mathrm{D}}\right)$ of trastuzumab and the various immunoconjugates for HER2, murine Fc $\gamma$ RI, and human Fc $\gamma$ RI were determined via SPR on a Biacore T200 (GE Healthcare). With respect to the former, SPR was performed using the immunoconjugate ( $n=3$ per construct) as the ligand captured on a Protein A sensor chip (29-1275-56; GE Healthcare). Subsequently, purified recombinant human HER2 protein (HE2-H822R; Acro Biosystems) was used as the analyte and injected over the sensor chip at a flow rate of $5 \mu \mathrm{L} / \mathrm{min}$. Next, the binding buffer (HEPES-buffered saline supplemented with ethylenediaminetetraacetic acid [EDTA] and polysorbate 20 [HBS-EP+]) was flowed over the sensor chip for $15 \mathrm{~min}$ at a rate of $5 \mu \mathrm{L} / \mathrm{min}$ to allow for the dissociation of HER2 from chip-bound immunocomplexes, and the regeneration buffer (10 mM Glycine- $\mathrm{HCl} \mathrm{pH} \mathrm{2.0)} \mathrm{was} \mathrm{passed} \mathrm{over} \mathrm{the} \mathrm{chip} \mathrm{surface}$ for $1 \mathrm{~min}$ at a flow rate of $5 \mu \mathrm{L} / \mathrm{min}$. HBS-EP+ buffer was then flowed $(5 \mu \mathrm{L} / \mathrm{min})$ over the chip for 2 min to stabilize the Protein A chip surface before the injection of the next sample.

With respect to human and murine Fc $\gamma \mathrm{RI}$, a histidine-tagged variant of recombinant human Fc $\gamma$ RI (huFc $\gamma$ RI) (500238; NovoPro Labs) was captured as the ligand on a Series S sensor chip NTA (BR-100532; GE Healthcare). To this end, the NTA chip was charged with nickel ions $\left(\mathrm{Ni}^{2+}\right)$ by performing a low sample consumption injection of $0.5 \mathrm{mM} \mathrm{NiCl}$ from the NTA Reagent kit (28-9950-43; GE 
Healthcare) for $60 \mathrm{~s}$ at a flow rate of $10 \mu \mathrm{L} / \mathrm{min}$, followed by an extra wash with HEPES-buffered saline supplemented with polysorbate 20 (HBS-P+ buffer) containing $3 \mathrm{mM}$ EDTA. Next, a $2 \mathrm{nM}$ solution of huFc $\gamma \mathrm{RI}$ in running buffer (HBS-P+ buffer containing $50 \mu \mathrm{M}$ EDTA) was injected over flow-cell 2 for $60 \mathrm{~s}$ at a flow rate of $10 \mu \mathrm{L} / \mathrm{min}$. Highperformance injections of various concentrations of the immunoconjugates were performed over flow-cells 1 and 2 for $300 \mathrm{~s}$ at a flow rate of 30 $\mu \mathrm{L} / \mathrm{min}$. To shorten the length of the SPR run without compromising the analysis of the bimolecular interaction, the dissociation of the immunoconjugates from the huFc $\gamma$ RI captured on the NTA chip was assessed over 2 different lengths of time: $900 \mathrm{~s}$ for the highest concentration of analyte and $300 \mathrm{~s}$ for all other concentrations. Finally, the surface of the flow-cells on the NTA chip was regenerated using a $60 \mathrm{~s}$ injection of $350 \mathrm{mM}$ EDTA (NTA Reagent kit) at a flow rate of $30 \mu \mathrm{L} / \mathrm{min}$, followed by an extra wash using the running buffer. A similar experimental set up was used to evaluate the interaction between the various trastuzumab immunoconjugates and murine $\mathrm{Fc} \gamma \mathrm{RI}(\mathrm{mFc} \gamma \mathrm{RI})$, though the dissociation of the immunoconjugates was evaluated over a shorter time window of 180 $\mathrm{s}$ because $\mathrm{mFc} \gamma \mathrm{RI}$ has 2 binding sites for human and humanized IgG1 and has been shown to display biphasic binding with these biomolecules.

The BIAcore T200 evaluation software was used to analyze the kinetic data, and the 1:1 Langmuir binding fit (refractive index set to $0)$ was used to derive the kinetic constants.

\section{Statistical Analysis}

The statistical analyses were performed using GraphPad Prism 7.0 software (GraphPad Software). All data are expressed as mean \pm SD. When applicable, statistical differences were analyzed by an unpaired, 2tailed Student $t$ test (with a Welch's correction when mentioned) and the 1way ANOVA for the comparison of more than 2 groups. Differences at the 95\% confidence level $(P<0.05)$ were considered statistically significant.

\section{Data Availability}

The datasets generated and analyzed during this study as well as detailed experimental procedures are readily available from the corresponding author upon request.

\section{RESULTS AND DISCUSSION}

\section{Synthesis and Characterization}

The HER2-targeting antibody trastuzumab was chosen as a model IgG1 because it is robust, well characterized, and easily

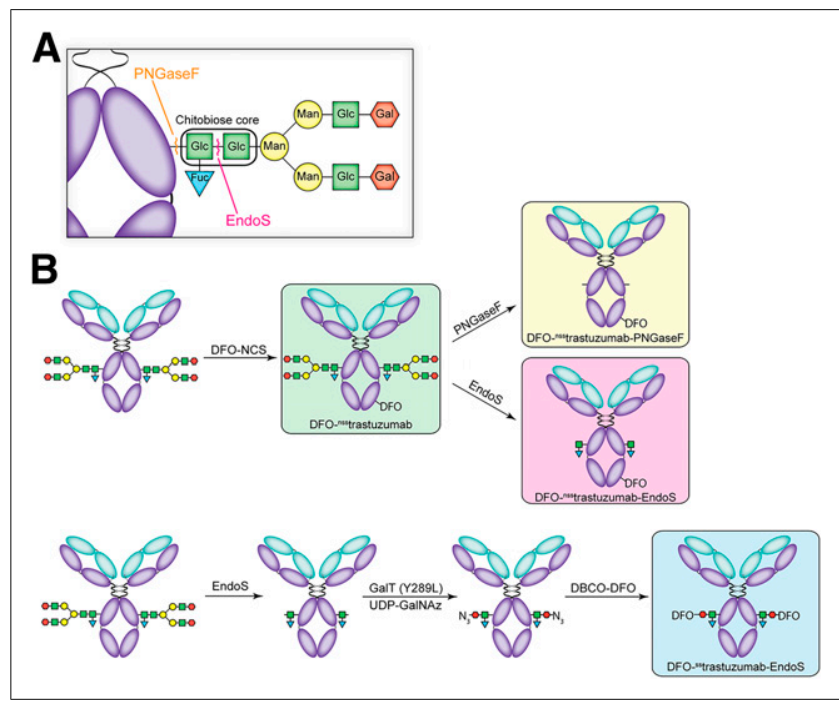

FIGURE 2. Preparation of the trastuzumab immunoconjugates. (A) Magnified view of cleavage sites for PNGaseF and EndoS. (B) Scheme of synthesis of the non-site-specifically and site-specifically modified immunoconjugates.

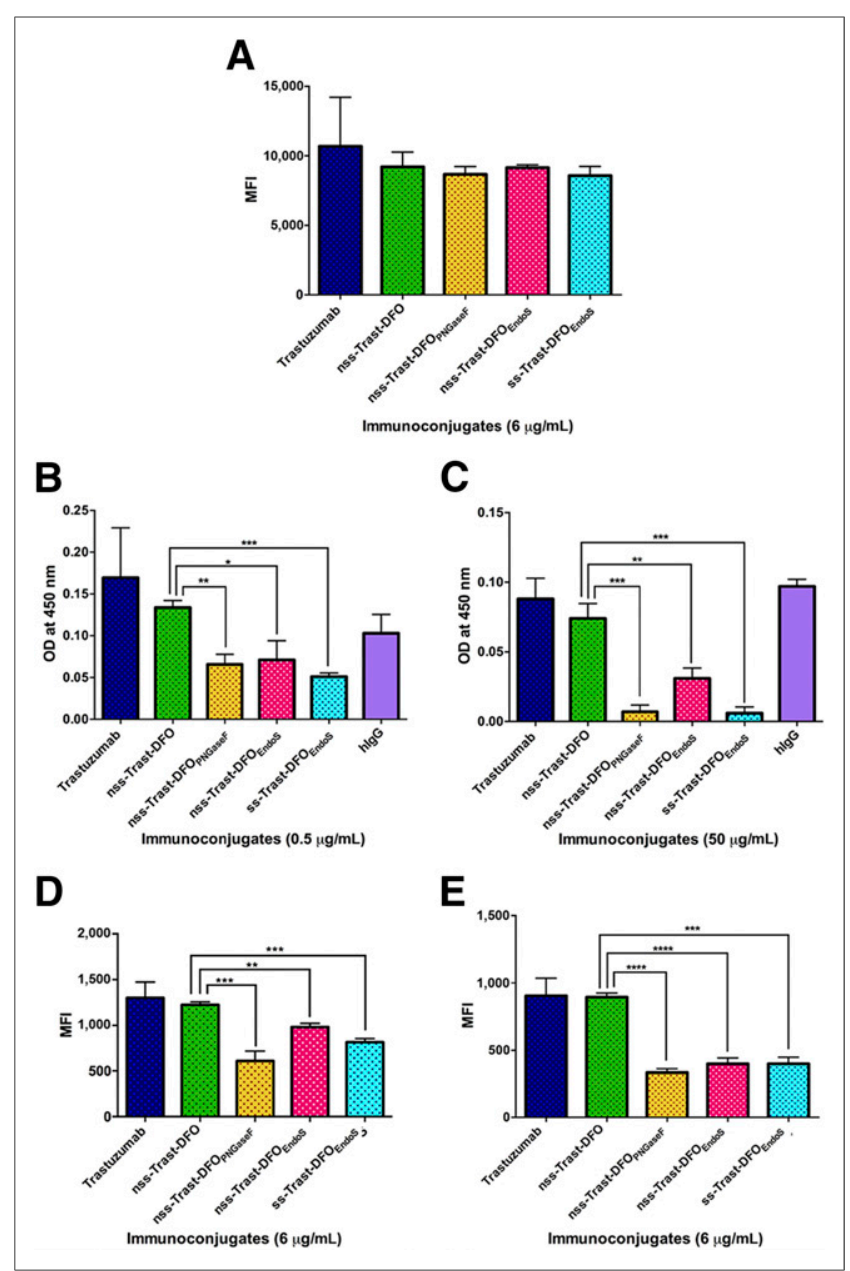

FIGURE 3. Flow cytometry and ELISA analyses of DFO-bearing immunoconjugates. (A) Flow cytometry with BT474 cells to verify HER2-binding. (B) ELISA of the immunoconjugates $(0.5$ or $50 \mu \mathrm{g} / \mathrm{mL})$ with huFcyRI $(10 \mu \mathrm{g} / \mathrm{mL})$ and $\mathrm{mFcyRI}(10 \mu \mathrm{g} / \mathrm{mL})$. (C) Flow cytometry of the immunoconjugates with human U937 macrophages and mouse RAW 264.7 macrophages. Data represent mean $\pm \mathrm{SD}$; each immunoconjugate was tested in triplicate (distinct samples). MFI = mean fluorescence intensity. Data were analyzed by unpaired, 2-tailed Student $t$ test: ${ }^{\star} P<0.05,{ }^{\star \star} P<0.01,{ }^{* \star \star} P<0.001,{ }^{* \star \star \star} P<0.0001$.

available. Furthermore, trastuzumab has been shown to recruit immune effector cells via interactions with $\mathrm{Fc} \gamma \mathrm{R}$ as part of its therapeutic mechanism (24). ${ }^{89} \mathrm{Zr}$ was used as the radionuclide due to its residualizing properties and its physical half-life of approximately $3.3 \mathrm{~d}$, which is ideally suited to complement the biologic half-life of IgG1. Finally, DFO was chosen as the chelator, as it is the "gold standard" chelator for ${ }^{89} \mathrm{Zr}$ in both the laboratory and the clinic (25).

The first step in the investigation was the modification of trastuzumab with DFO. To this end, we pursued 2 different approaches: nonsite-specific and site-specific bioconjugation. The former was achieved via the random-or non-site-specific-reaction of an isothiocyanate-bearing variant of DFO ( $p$-SCN-Bn-DFO) with the lysines of the antibody (17). The resulting immunoconjugate-DFO- ${ }^{\text {nss }}$ trastuzumab-has an average of 1.2 DFO per antibody as estimated by MALDI-TOF. This immunoconjugate was then deglycosylated using 2 different enzymes (Figs. 2A and 2B). The first, peptide: $N$-glycosidase F (PNGaseF), cleaves the entire 
glycan chain linked to asparagine at position 297 (Q297), creating a completely deglycosylated immunoconjugate: DFO-nss trastuzumab-PNGaseF. The second, EndoS, hydrolyses the chitobiose core of the asparagine-linked glycans, producing a partially deglycosylated immunoconjugate: DFO- ${ }^{\text {nss }}$ trastuzumab-EndoS (26).

We also created a site-specifically modified, DFO-bearing immunoconjugate via the chemoenzymatic modification of the glycans (Fig. 2B) (27). To this end, trastuzumab was first treated with EndoS and then incubated with the promiscuous galactosyltransferase GalT(Y289 L) and the monosaccharide UDP-GalNAz to incorporate azide-bearing sugars into the truncated glycans. Subsequently, DFO was introduced via the strain promoted alkyne-azide cycloaddition between dibenzocyclooctyne (DBCO)DFO and the azide-bearing glycans, and a degree-of-labeling of 1.3 DFO/monoclonal antibody was estimated via MALDI-TOF (Supplemental Fig. 1; supplemental materials are available at http://jnm.snmjournals.org). Critically, in all 3 cases, the complete removal of glycans was monitored using gel electrophoresis under reducing conditions and confirmed via both a Lens culinaris agglutinin blot and MALDI-TOF (Supplemental Figs. 1-3).

\section{In Vitro Characterization}

The first step in the in vitro evaluation of the immunoconjugates was the assessment of their binding to HER2. SPR revealed that all 4 immunoconjugates exhibit binding affinities and on- and offrates with HER2 comparable to that of native trastuzumab (Supplemental Fig. 4 and Supplemental Table 1). Flow cytometry with HER2-positive BT474 cells subsequently confirmed that the immunoreactivity of all 4 immunoconjugates is unperturbed (Fig. 3A; Supplemental Fig. 5).

Next, the interactions between the immunoconjugates and Fc $\gamma$ RI were probed via SPR. Several groups have shown that human $\mathrm{IgG}_{1}$ exhibit similar interaction patterns with-though different affinities for-human and murine Fc receptors $(28,29)$. Since the in vivo behavior of our immunoconjugates would be tested in mice, we decided to investigate their binding to murine Fc $\gamma R I$ (mFc $\gamma R I)$ as well as human Fc $\gamma$ RI (huFc $\gamma R I)$. Taken together, the SPR data clearly indicate that deglycosylation negatively affects the interaction between the immunoconjugates and Fc $\gamma R I$, though the phenomenon is more pronounced with huFc $\gamma \mathrm{RI}$ than mFc $\gamma$ RI (Fig. 4; Supplemental Fig. 6, Supplemental Tables $2-5)$. DFO-nss trastuzumab possesses $\mathrm{K}_{\mathrm{D}}$, on-rate, and off-rate values with huFc $\gamma$ RI comparable to unmodified trastuzumab (Fig. 4). In contrast, DFO-nss trastuzumab-PNGaseF exhibits a 20-fold reduction in $\mathrm{K}_{\mathrm{D}}$ for huFc $\gamma \mathrm{RI}$ compared with native trastuzumab as well as a dramatically accelerated off-rate. Interestingly, the 2 EndoS-modified immunoconjugates-DFO-nss trastuzumab-EndoS and DFO-sstrastuzumab-EndoS-present intermediate cases, with $\mathrm{K}_{\mathrm{D}}$ values and off-rates between that of native trastuzumab and DFO-nsstrastuzumab-PNGaseF. The impact of deglycosylation on the huFc $\gamma \mathrm{RI}$ binding of the immunoconjugates is reinforced by the dramatic decreases in the half-life of the interaction between the 2 components: DFO- ${ }^{\text {nss }}$ trastuzumab boasts a half-life of $86.1 \pm$ 9.6 min with huFc $\gamma \mathrm{RI}$, a value that decreases to $15.4 \pm 0.5$ for

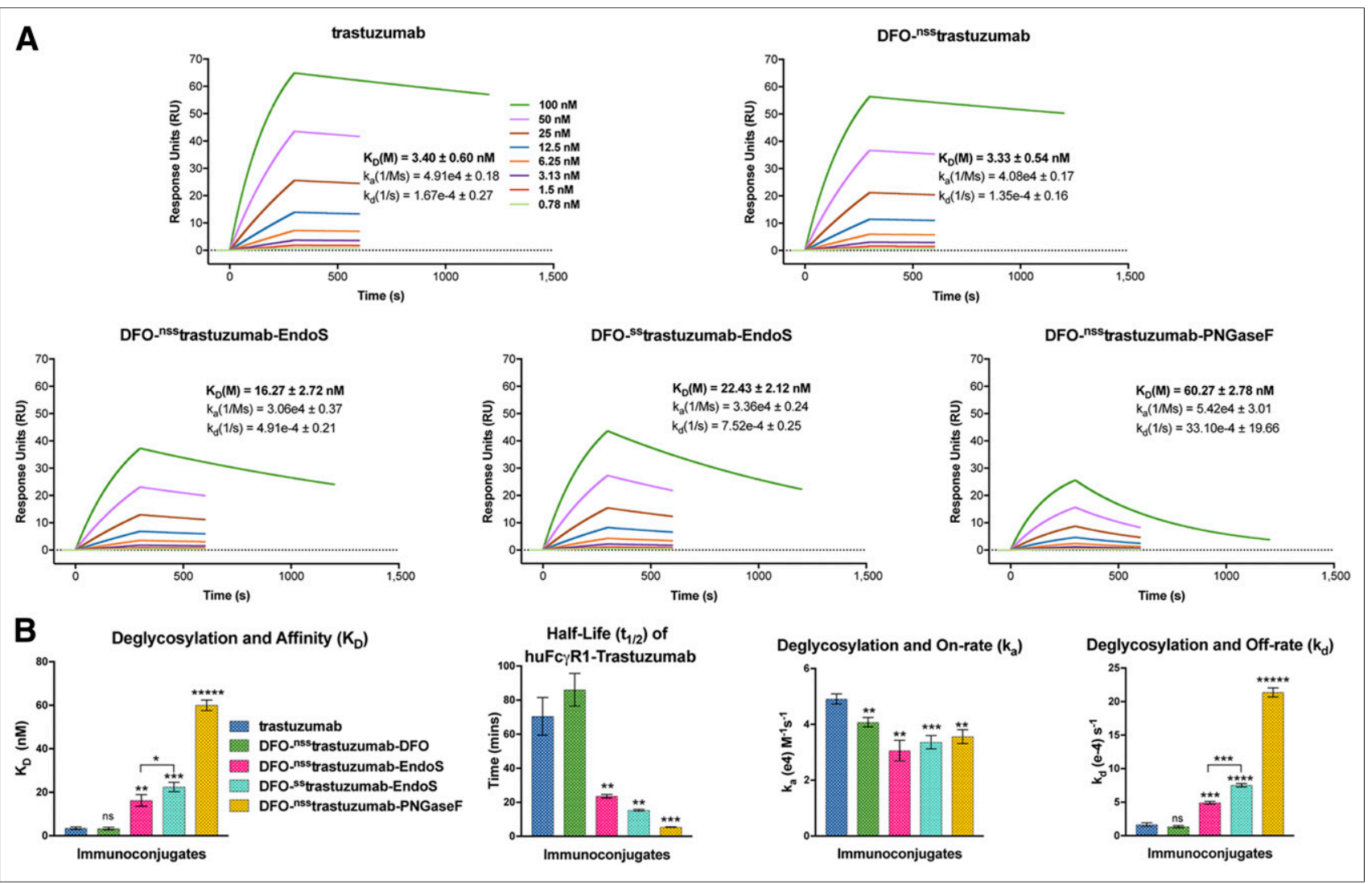

FIGURE 4. SPR analysis of interaction between the immunoconjugates and recombinant huFcyRI. (A) Binding affinity and kinetic rate constants for each immunoconjugate are shown in sensorgram plots. (B) Bar graphs illustrating correlation between deglycosylation and binding affinity ( $\left.\mathrm{K}_{\mathrm{D}}\right)$, half-

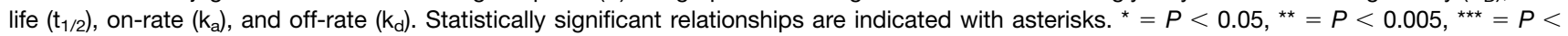
$0.0005,{ }^{\star \star \star *}=P<0.00005,{ }^{\star \star \star \star \star}=P<0.000005$. 
DFO- ${ }^{\text {ss }}$ trastuzumab-EndoS and to $5.4 \pm 0.2$ for DFO- ${ }^{\text {nss }}$ trastuzumab-PNGaseF.

Moving on to $\mathrm{mFc} \gamma \mathrm{RI}$, native trastuzumab displays an approximately 10-fold-lower binding affinity $(\sim 37 \mathrm{nM})$ for $\mathrm{mFc} \gamma \mathrm{RI}$ than for huFc $\gamma \mathrm{RI}(\sim 3 \mathrm{nM})$, a result that is in agreement with the wider literature (Supplemental Fig. 6; Supplemental Tables 4 and 5) (13,28-31). Yet still, the EndoS-treated immunoconjugates exhibit decreased $K_{D}$ values and accelerated off-rates compared with native trastuzumab. Surprisingly, DFO- ${ }^{\text {nss }}$ trastuzumab-PNGaseF displays a $K_{D}$ similar to that of unmodified trastuzumab. Yet it is important to note that this value is not representative of the true behavior of the immunoconjugate with $m F c \gamma R I$. Since $K_{D}=k_{d} / k_{a}$, this seemingly strong binding affinity is most likely the product of a high off-rate and an equally fast on-rate. In light of this, the halflives of the immunoconjugates with $\mathrm{mFc} \gamma \mathrm{RI}$ likely provide the most complete picture of their interaction with the receptor. The half-lives of all 5 constructs with $\mathrm{mFc} \gamma \mathrm{RI}$ are significantly shorter than the analogous values for their interaction with huFc $\gamma R$ I. However, the values for the 3 deglycosylated immunoconjugates are

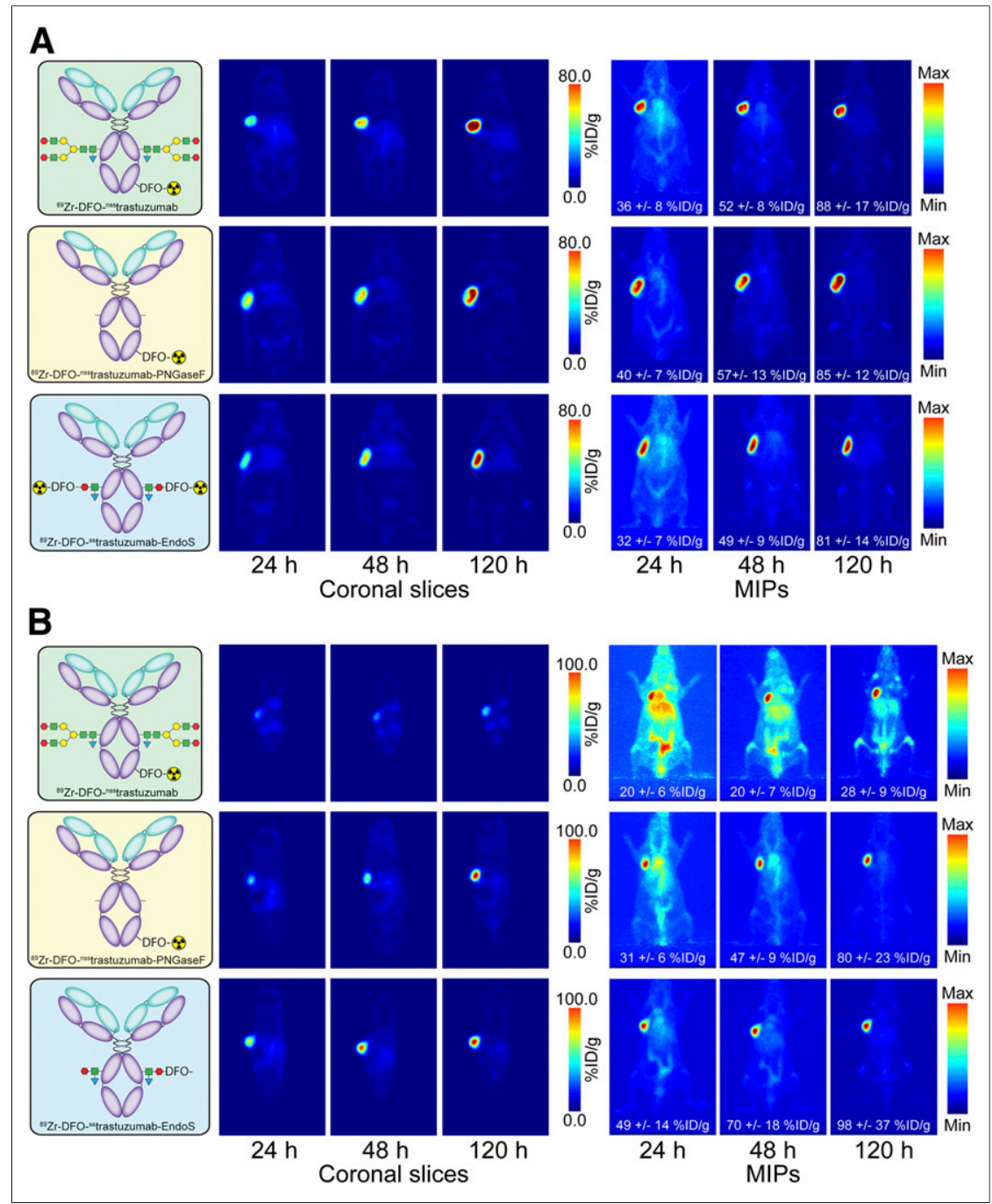

FIGURE 5. PET imaging in nude and NSG mice. Planar (left) and maximum-intensity-projection (MIP, right) PET images of nude (A) and NSG (B) mice bearing subcutaneous BT474 xenografts at 24,48 , and $120 \mathrm{~h}$ after injection. Values in white represent tumoral activity concentrations in $\% \mathrm{ID} / \mathrm{g} \pm \mathrm{SD}$ as determined via region-of-interest analysis $(n=4)$. significantly shorter than those of DFO-nsstrastuzumab and native trastuzumab, with DFO-nss trastuzumab-PNGaseF having the shortest half-life of all.

These relationships between deglycoslation and Fc $\gamma$ RI binding are reinforced by ELISA and in vitro binding assays. ELISA data with huFc $\gamma$ RI clearly indicate that DFO- ${ }^{\text {nss }}$ trastuzumab-PNGaseF, DFO- ${ }^{\text {nss }}$ trastuzumab-EndoS, and DFO- ${ }^{\mathrm{ss}}$ trastuzumab-EndoS all exhibit decreased binding to huFc $\gamma$ RI compared with DFO- ${ }^{n s}$ trastuzumab and native trastuzumab (Fig. 3B; Supplemental Fig. 7). Significantly higher concentrations of the immunoconjugates (50 $\mu \mathrm{g} / \mathrm{mL}$ ) had to be used to evaluate $\mathrm{mFc} \gamma \mathrm{RI}$ binding via ELISA, though similar patterns were uncovered: the deglycosylated constructs all have attenuated binding to $\mathrm{mFc} \gamma \mathrm{RI}$ compared with the parent constructs (Fig. 3B). Next, because both huFc $\gamma$ RI and $\mathrm{mFc} \gamma \mathrm{RI}$ can be found on the surface of macrophages, a murine macrophage cell line (RAW 264.7) as well as a human lymphoma cell line differentiated into mature macrophages (U937) were selected for flow cytometry experiments (Fig. 3C; Supplemental Figs. 8 and 9) (14,32). As one might expect based on the ELISA and SPR data, DFO-nss trastuzumabPNGaseF, DFO-nsstrastuzumab-EndoS, and DFO- ${ }^{\text {ss }}$ trastuzumab-EndoS all displayed decreased binding to both murine and human macrophages.

\section{In Vivo Behavior}

The next step in the investigation was interrogating the in vivo behavior of the deglycosylated immunoconjugates. Given the exigencies of in vivo preclinical work, 2 of the immunoconjugates bearing modified glycans-DFO- ${ }^{\text {nss }}$ trastuzumab-PNGaseF and DFO- ${ }^{\text {ss }}$ trastuzumab-EndoS-were chosen for in vivo evaluation alongside their more traditional analog, DFO-nsstrastuzumab. Our logic here was simple: the former possesses the shortest half-lives with both huFc $\gamma \mathrm{RI}$ and $\mathrm{mFc} \gamma \mathrm{RI}$, and the latter offers the dual advantages of site-specific bioconjugation and attenuated binding to huFc $\gamma \mathrm{RI}$ and $\mathrm{mFc} \gamma \mathrm{RI}$. These immunoconjugates were radiolabeled with ${ }^{89} \mathrm{Zr}$ via standard protocols, producing a trio of radioimmunoconjugates in greater than $95 \%$ radiochemical yield and greater than $99 \%$ radiochemical purity with specific activities of $81.4-99.9 \mathrm{MBq} / \mathrm{mg}$. The stability of the radioimmunoconjugates was tested in human serum at $37^{\circ} \mathrm{C}$, and all 3 demonstrated more than $85 \%$ stability after 7 d (Supplemental Fig. 10).

We conducted the initial in vivo evaluation of these radioimmunoconjugates in athymic nude mice because they are the most commonly used immunodeficient strain used for xenograft models of human cancer. Furthermore, nude mice possess immune effector cells that express the murine Fc $\gamma$ RI receptor, including macrophages, natural killer cells, and dendritic cells (33). The in vivo performance of the radioimmunoconjugates was evaluated via PET imaging experiments as 
well as biodistribution studies in mice bearing subcutaneous HER2expressing BT474 human breast cancer xenografts. PET images were collected at 24,48 , and $120 \mathrm{~h}$ after the injection of the radioimmunoconjugates $(7.0-7.8 \mathrm{MBq}, 70-80 \mu \mathrm{g})$. At the earliest time point $(24 \mathrm{~h})$, the images revealed that all 3 radioimmunoconjugates accumulated in the HER2-positive tumors. In addition, ${ }^{89} \mathrm{Zr}$ DFO- ${ }^{\text {nss }}$ trastuzumab, ${ }^{89} \mathrm{Zr}$-DFO- ${ }^{\text {ss }}$ trastuzumab-EndoS, and ${ }^{89} \mathrm{Zr}$ DFO-nsstrastuzumab-PNGaseF all produced background signal in the heart and liver, the latter creating the lowest activity concentrations in these tissues (Fig. 5A). Over the course of the experiment, the background uptake decreased for all 3 radioimmunoconjugates in favor of higher tumoral activity concentrations. The biodistribution data tell a similar story (Supplemental Fig. 11 and Supplemental Table 6). Taken together, these data clearly suggest that deglycosylation with PNGaseF and site-specific modification using EndoS had produced radioimmunoconjugates with in vivo behavior comparable to that of traditional ${ }^{89} \mathrm{Zr}$-DFO-nsstrastuzumab.

Our findings in athymic nude mice did little to support our initial hypothesis. However, Overdijk et al. have previously cautioned that the activation of cellular immune effector functions by hIgG1 might be underestimated in mice due to differences between mFc $\gamma R I$ and huFc $\gamma R I(28,29)$. Armed with this knowledge, we sought a more advantageous model to highlight the impact of deglycosylation on the biodistribution of our radioimmunoconjugates. To this end, we turned to a highly immunodeficient strain: NOD scid

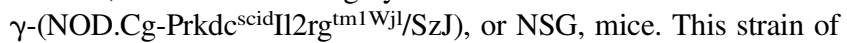
mice lacks endogenous immunoglobulins that can compete with radioimmunoconjugates for unoccupied $\mathrm{mFc} \gamma \mathrm{RI}$ on myeloid cells such as monocytes, neutrophils, and eosinophils, as well as tissueresident macrophages in the liver, spleen, and bone (18).

For these experiments, NSG mice bearing subcutaneous HER2expressing BT474 xenografts were injected via the tail vein with the 3 radioimmunoconjugates $(6.5-7.4 \mathrm{MBq}, 60-80 \mu \mathrm{g})$ and imaged after 24, 48, and $120 \mathrm{~h}$ (Fig. 5B). PET imaging with all

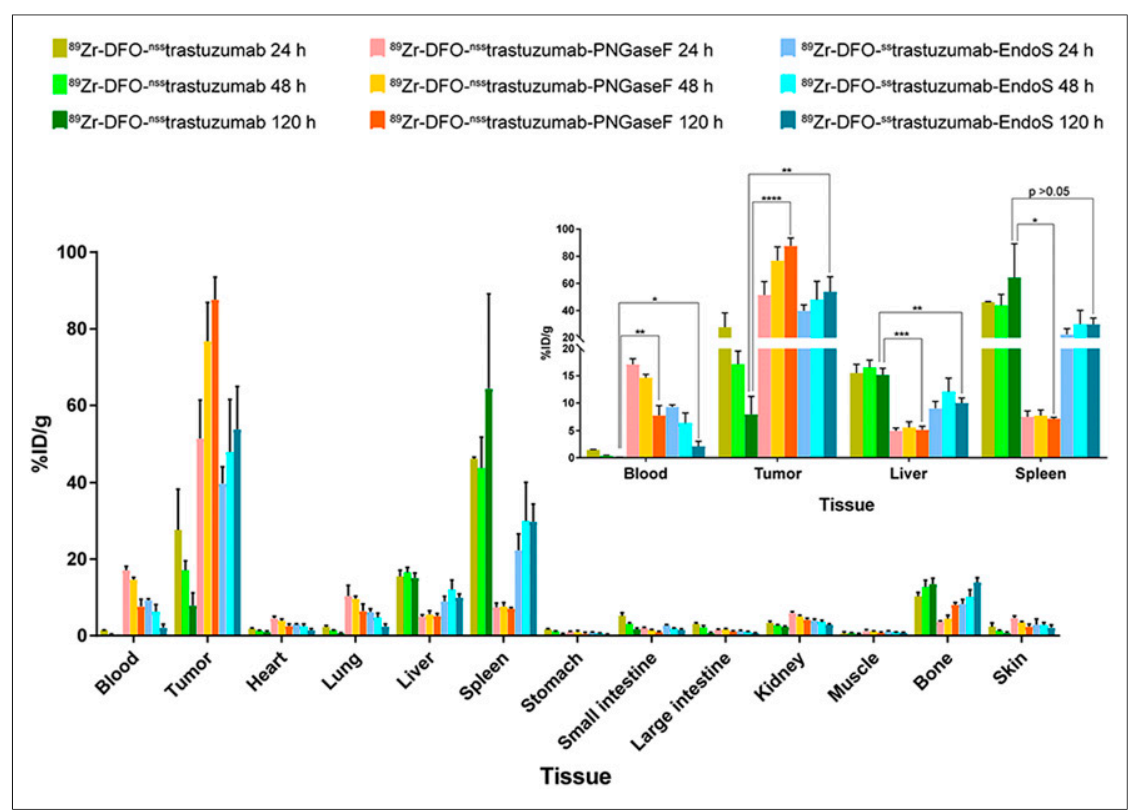

FIGURE 6. In vivo biodistribution profile for ${ }^{89} \mathrm{Zr}$-DFO-nsstrastuzumab, ${ }^{89} \mathrm{Zr}$-DFO-nsstrastuzumab-PNGaseF, and ${ }^{89} \mathrm{Zr}$-DFO-sstrastuzumab-EndoS at 24,48 , and $120 \mathrm{~h}$ after injection in NSG mice bearing subcutaneous BT474 xenografts. Data represent mean $\pm \mathrm{SD}, n=4$. Data were analyzed by 1 -way ANOVA: ${ }^{\star} P<0.05,{ }^{\star \star} P<0.01,{ }^{\star \star \star} P<0.001,{ }^{\star \star \star \star} P<0.0001$.
3 radioimmunoconjugates clearly delineated the BT474 xenografts as early as $24 \mathrm{~h}$ after injection. However, besides high-activity concentrations in the heart and blood vessels, a marked difference was apparent in the images produced by the 3 radioimmunoconjugates: ${ }^{89} \mathrm{Zr}$-DFO-nsstrastuzumab produced very-high-activity concentrations in the liver, whereas the pair of radioimmunoconjugates with modified glycans yielded far lower uptake in the organ. By $120 \mathrm{~h}$ after injection, the tumor was the most prominent feature in the scans collected with ${ }^{89} \mathrm{Zr}$-DFO- ${ }^{\text {nss }}$ trastuzumab-PNGaseF and ${ }^{89} \mathrm{Zr}$-DFO- ${ }^{\mathrm{ss}}$ trastuzumab-EndoS, whereas the images produced by ${ }^{89} \mathrm{Zr}$-DFO-nss trastuzumab-DFO show dramatically lower tumoral uptake as well as significant off-target activity concentrations in the liver and bone. Interestingly, over the course of the experiment, the clearance of ${ }^{89} \mathrm{Zr}$-DFO- ${ }^{\mathrm{nss}}$ trastuzumab-PNGaseF from the heart and blood vessels appeared slower than either of the 2 other radioimmunoconjugates, an observation consistent with the idea of an "immune-silent" radioimmunoconjugate.

The differences between the in vivo performance of ${ }^{89} \mathrm{Zr}$ DFO-nss trastuzumab-PNGaseF and ${ }^{89} \mathrm{Zr}$-DFO-nss trastuzumab were confirmed by biodistribution studies (Fig. 6). At $120 \mathrm{~h}$ after injection, the tumoral activity concentration produced by ${ }^{89} \mathrm{Zr}$-DFO-nsstrastuzumab-PNGaseF $(87.7 \pm 5.9$ percentage injected dose per gram [\% ID/g]) was significantly higher than that created by ${ }^{89} \mathrm{Zr}-\mathrm{DFO}-$ ${ }^{n s s}$ trastuzumab (7.9 $\pm 3.3 \%$ ID/g; Fig. 6; Supplemental Table 7). Simultaneously, the activity concentrations in the liver and spleen of the NSG mice injected with the fully deglycosylated immunoconjugate were reduced by 3- and 9-fold, respectively. These 2 trends yield dramatically improved tumor-to-liver and tumor-to-spleen activity concentration ratios for ${ }^{89} \mathrm{Zr}$-DFO- ${ }^{\text {nss }}$ trastuzumab-PNGaseF $\left(17.0 \pm 2.5\right.$ and $12.3 \pm 0.9$, respectively) compared with ${ }^{89} \mathrm{Zr}-\mathrm{DFO}-$ ${ }^{\text {nss }}$ trastuzumab $(0.5 \pm 0.2$ and $0.1 \pm 0.1$, respectively) (Supplemental Table 7). However, the persistence of the deglycosylated radioimmunoconjugate in the blood yielded lower tumor-to-blood activity concentration ratios for ${ }^{89} \mathrm{Zr}$-DFO- ${ }^{\text {nss }}$ trastuzumab-PNGaseF compared with ${ }^{89} \mathrm{Zr}$-DFO-nss trastuzumab: $11.4 \pm 2.8$ versus $112.7 \pm 67.7$ at $120 \mathrm{~h}$ after injection (Supplemental Table 8). Likewise, highly perfused organs such as the heart and lungs also exhibited decreased tumor-to-background activity concentration ratios with ${ }^{89} \mathrm{Zr}$-DFO- ${ }^{\text {nss }}$ trastuzumab-PNGaseF, almost certainly due to the persistence of the radioimmunoconjugate in the blood. ${ }^{89} \mathrm{Zr}$ DFO-sstrastuzumab-EndoS proved to be an intermediate case. Briefly, the site-specifically modified immunoconjugate showed higher tumoral uptake values (e.g., $53.9 \pm 11.0 \% \mathrm{ID} / \mathrm{g}$ at $120 \mathrm{~h}$ after injection) than ${ }^{89} \mathrm{Zr}$-DFO${ }^{n s s}$ trastuzumab but higher activity concentrations in the liver and spleen than ${ }^{89} \mathrm{Zr}$-DFO-nss trastuzumab-PNGaseF.

Ultimately, whereas NSG mice allow us to demonstrate the benefit of using deglycosylated "immune-silent" antibodies for immuno-PET, the NSG platform is an exaggerated immunodeficient model with little clinical relevance. To wit, whereas nude mice have serum total IgG titers greater than 40 $\mu \mathrm{g} / \mathrm{mL}$, the IgG titers in NSG mice are almost indetectable (18). In contrast, human patients possess endogenous immunoglobulins that 
occupy huFc $\gamma \mathrm{RI}$ on immune effector cells. This, of course, has the potential to abrogate the benefits provided by immune-silent radioimmunoconjugates that we have seen in the NSG mice.

Having evaluated our immunoconjugates in 2 different immunodeficient strains of mice, we were eager to test our hypothesis in a more clinically relevant model. To this end, we next characterized the in vivo behavior of our radioimmunoconjugates in humanized mice. Specifically, we used NSG mice that were sublethally irradiated at 3 wk after birth and subsequently reconstituted with human hematopoietic stem cells derived from cord blood to express a functional human immune system, including human B cells, T cells, NK cells, dendritic cells, and monocytes $(34,35)$. The last entry in this list is particularly important, as monocytes are the primary human immune system cells expressing Fc $\gamma$ RI (14). Importantly, a titration of the serum from these mice confirmed the presence of endogenous human $\operatorname{IgG}(202 \pm 67 \mu \mathrm{g} / \mathrm{mL})$ in levels consistent with published data on the strain (Supplemental Fig. 12) $(34,36)$.

Given the high cost of humanized mice, we chose to compare a pair of radioimmunoconjugates: the control ${ }^{89} \mathrm{Zr}$-DFO- ${ }^{\text {nss }}$ trastuzumab and the deglycosylated ${ }^{89} \mathrm{Zr}$-DFO- ${ }^{\text {nss }}$ trastuzumab-PNGaseF. To this end, humanized NSG mice (huNSG) bearing subcutaneous HER2-positive BT474 human breast cancer xenografts were injected with the radioimmunoconjugates (6.8-7.4 MBq), and PET imaging was performed after 24,48 , and $120 \mathrm{~h}$. Images obtained

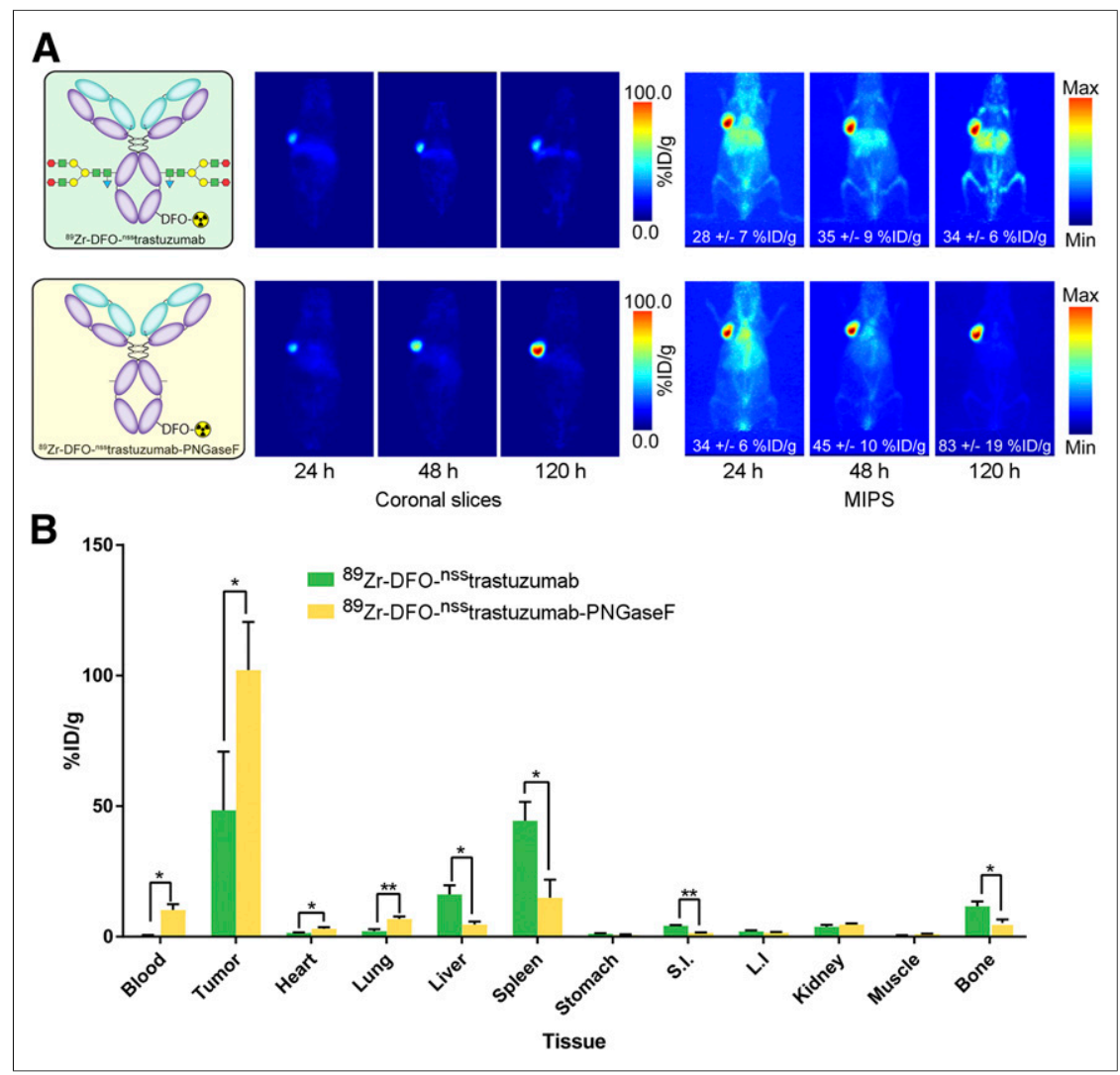

FIGURE 7. In vivo results in humanized NSG mice. (A) Planar (left) and maximum-intensityprojection (MIP, right) PET images of humanized NSG mice bearing subcutaneous BT474 xenografts at 24,48 , and $120 \mathrm{~h}$ after injection. Values in white represent tumoral activity concentrations in $\% \mathrm{ID} / \mathrm{g} \pm \mathrm{SD}$ as determined via region-of-interest analysis $(n=4)$. (B) In vivo biodistribution profile for ${ }^{89} \mathrm{Zr}$-DFO-nsstrastuzumab and ${ }^{89} \mathrm{Zr}$-DFO-nsstrastuzumab-PNGaseF at $120 \mathrm{~h}$ after injection in humanized NSG mice bearing BT474 tumors. Data represent mean $\pm \mathrm{SD}, n=4$. Data were analyzed by unpaired, 2-tailed Student $t$ test with a Welch's correction: ${ }^{\star} P<0.05,{ }^{\star \star} P<0.01$. with both radioimmunoconjugates display a clear delineation of tumors as early as $24 \mathrm{~h}$ after injection, though the tumoral activity concentrations produced by ${ }^{89} \mathrm{Zr}$-DFO- ${ }^{\text {nss }}$ trastuzumab-PNGaseF are far higher than those produced by ${ }^{89} \mathrm{Zr}$-DFO- ${ }^{\text {nss }}$ trastuzumab (Fig. 7A). However, the patterns of nonspecific uptake created by the 2 immunoconjugates were dramatically different. ${ }^{89} \mathrm{Zr}$ DFO- ${ }^{n s}$ trastuzumab-PNGaseF exhibited relatively high activity concentrations in the heart throughout the experiment but very low accumulation in any other nontarget tissues. ${ }^{89} \mathrm{Zr}$-DFO- ${ }^{\text {nss }}$ trastuzumab, in contrast, produced lower activity concentrations in the heart and blood vessels but far higher levels of uptake in the liver, spleen, and bones. These differences are particularly striking in the maximum-intensity projections collected $120 \mathrm{~h}$ after injection. For ${ }^{89} \mathrm{Zr}$-DFO-nss trastuzumab-PNGaseF, the only visible feature is the tumor. For ${ }^{89} \mathrm{Zr}$-DFO- ${ }^{\text {nss }}$ trastuzumab, the tumor boasts the highest uptake, but the liver, spleen, and bones are prominent as well.

The differences between the in vivo performances of the 2 radioimmunoconjugates were further illustrated by a biodistribution study performed $120 \mathrm{~h}$ after injection (Fig. 7B; Supplemental Fig. 13, Supplemental Table 9). The deglycosylated radioimmunoconjugate $\left({ }^{89} \mathrm{Zr}\right.$-DFO-nss trastuzumab-PNGaseF) produced nearly double the tumoral activity concentration created by ${ }^{89} \mathrm{Zr}$-DFO- ${ }^{\text {nss }}$ trastuzumab: $102.1 \pm 18.5 \% \mathrm{ID} / \mathrm{g}$ versus $48.4 \pm 22.5 \% \mathrm{ID} / \mathrm{g}$. Furthermore,

${ }^{89} \mathrm{Zr}$-DFO-nsstrastuzumab-PNGaseF yielded much lower uptake in the liver, spleen, and bone. ${ }^{89} \mathrm{Zr}$-DFO-nsstrastuzumab-PNGaseF produced much higher activity concentrations in the blood as well as highly perfused organs such as the lungs and heart. This observation can be attributed to the persistence of the radioimmunoconjugate in systemic circulation, behavior in line with the notion of an "immune-silent" radioimmunoconjugate. Taken together, these in vivo data underscore the improved performance of the deglycosylated radioimmunoconjugate relative to its more traditional, fully glycosylated analog. Indeed, ${ }^{89} \mathrm{Zr}-\mathrm{DFO}-$ nss trastuzumab-PNGaseF produced high tumor-to-background activity concentration ratios and excellent immuno-PET images despite the presence of a fully reconstituted human immune system and the endogeneous IgGs in huNSG mice. Although ${ }^{89} \mathrm{Zr}$ DFO-nsstrastuzumab enabled the visualization of tumor tissue, it was also subject to $\mathrm{Fc} /$ Fc $\gamma R$-mediated uptake in nontarget organs such as the liver, spleen, and bones.

Before we delve into the implications of this work, it is important to issue 4 caveats. First, further investigations into the generality of this phenomenon are certainly warranted. Second, although humanized mice were used as a surrogate to study the impact of the in vivo interaction between humanized IgG1 and the human immune system, it is important to note that this model has its limitations (37). Specifically, the titers of endogenous human IgGs in this mouse model are significantly lower than in 
immunocompetent mouse strains due to the lack of class switching from IgM to $\operatorname{IgG}$ isotypes in vivo. This might be attributed to the lack of B-cell maturation in the humanized mouse model due to the absence of functional lymph nodes, germinal centers, and follicular dendritic cells $(38,39)$. Third, physiology-based pharmacokinetic models suggest that $\mathrm{Fc} / \mathrm{Fc} \gamma \mathrm{R}$ interactions do not affect the in vivo biodistribution of antibody-based imaging agents and drugs due to the occupancy of Fc $\gamma \mathrm{R}$ sites by the preexisting pool of endogenous immunoglobulins (40). And finally, it is also important to note that nonspecific uptake of radioimmunoconjugates in the liver is not solely mediated by Fc $\gamma$ RI. To wit, aggregated antibodies are also routed to the liver, and in the presence of shed antigens, the formation of soluble immunocomplexes can be another cause of this offtarget signal. In these cases, deglycosylation may not be of any help for improving imaging quality.

Ultimately, we believe that this work could have an impact in both the laboratory and the clinic. These data clearly suggest that deglycosylation is an inexpensive, effective, and modular approach to improving the quality of immuno-PET images collected in preclinical models of cancer. Indeed, we believe that this strategy could be particularly useful in studies using highly immunodeficient mice or humanized mouse models. Furthermore, the benefits of deglycosylation can be coupled with the advantages of site-specific labeling using the EndoS-based bioconjugation strategy that we have described here.

The clinical implications of this work are admittedly less clear. Patients undergoing immuno-PET scans are routinely pre- or codosed with an excess of unlabeled target-specific antibody (e.g., $\sim 48 \mathrm{mg}$ of trastuzumab alongside $\sim 2 \mathrm{mg}$ of ${ }^{89} \mathrm{Zr}$-DFO-trastuzumab) (41). This practice consistently improves the contrast of images by decreasing the uptake of the radioimmunoconjugate in healthy nontarget organs, specifically the liver. If the root of this phenomenon lies in the ability of the unlabeled antibody to saturate Fc $\gamma$ RI binding sites in nontarget organs, the use of deglycosylated radioimmunoconjugates could offer a cheaper and easier means to the same ends, thereby enabling the more sensitive detection of metastases in the liver and other nontarget organs. On the other hand, as we have noted above, higher endogenous IgG levels may preclude the benefits of deglycosylation in most patients. In this case, deglycosylated radioimmunoconjugates could still prove valuable for immuno-PET in patients-including recent recipients of chemotherapy or radiation therapy - with low B-cell counts (42).

\section{CONCLUSION}

In this work, we have interrogated the role of $\mathrm{Fc} / \mathrm{Fc} \gamma \mathrm{RI}$ interactions on the in vivo performance of antibody-based PET imaging agents. Our findings suggest that the binding of humanized $\operatorname{IgG} 1$ by Fc $\gamma$ RI contributes to the uptake of radioimmunoconjugates in nontarget tissues during immuno-PET imaging. As a result, we hypothesized that attenuating these $\mathrm{Fc} / \mathrm{Fc} \gamma \mathrm{RI}$ interactions via the use of deglycosylated radioimmunoconjugates could be a viable strategy for decreasing unwanted uptake in healthy organs. We have demonstrated that deglycosylated radioimmunoconjugates not only exhibit decreased affinity for FcyRI but also produce both lower activity concentrations in nontarget organs and higher activity concentrations in tumor tissue in NSG and huNSG mice compared with a fully glycosylated analog. In the end, these findings suggest that deglycosylation is a facile, inexpensive, and versatile approach to the creation of "immune-silent" radioimmunoconjugates that could be more effective imaging agents than traditional radiolabeled antibodies in a wide variety of preclinical studies as well as certain clinical patient populations. We look forward to further exploring the implications of this finding for immuno-PET as well as interrogating the ramifications that these findings may have in the context of antibody-drug conjugates and therapeutic radioimmunoconjugates.

\section{DISCLOSURE}

The authors are grateful for the generous financial support of the National Institutes of Health (Brian M. Zeglis; R01CA204167) and the Tow Foundation (Sai Kiran Sharma). Services provided by the MSKCC Small-Animal Imaging Core Facility were supported in part by NIH grants R24 CA83084 and P30 CA08748. No other potential conflict of interest relevant to this article was reported.

\section{REFERENCES}

1. Wu AM. Antibodies and antimatter: the resurgence of immuno-PET. J Nucl Med. 2009;50:2-5.

2. Pyzik M, Rath T, Lencer WI, Baker K, Blumberg RS. FcRn: the architect behind the immune and nonimmune functions of $\mathrm{IgG}$ and albumin. J Immunol. 2015; 194:4595-4603.

3. Nimmerjahn F, Ravetch JV. Fcgamma receptors as regulators of immune responses. Nat Rev Immunol. 2008;8:34-47.

4. Sockolosky JT, Szoka FC. The neonatal Fc receptor, FcRn, as a target for drug delivery and therapy. Adv Drug Deliv Rev. 2015;91:109-124.

5. Pincetic A, Bournazos S, DiLillo DJ, et al. Type I and type II Fc receptors regulate innate and adaptive immunity. Nat Immunol. 2014;15:707-716.

6. Leelawattanachai J, Kwon KW, Michael P, Ting R, Kim JY, Jin MM. Side-byside comparison of commonly used biomolecules that differ in size and affinity on tumor uptake and internalization. PLoS One. 2015;10:e124440.

7. Hezareh M, Hessell AJ, Jensen RC, van de Winkel JGJ, Parren PWHI. Effector function activities of a panel of mutants of a broadly neutralizing antibody against human immunodeficiency virus type 1. J Virol. 2001;75:12161-12168.

8. Sondermann P, Szymkowski DE. Harnessing Fc receptor biology in the design of therapeutic antibodies. Curr Opin Immunol. 2016;40:78-87.

9. Raju TS, Briggs JB, Borge SM, Jones AJ. Species-specific variation in glycosylation of IgG: evidence for the species-specific sialylation and branch-specific galactosylation and importance for engineering recombinant glycoprotein therapeutics. Glycobiology. 2000;10:477-486.

10. Liu L. Antibody glycosylation and its impact on the pharmacokinetics and pharmacodynamics of monoclonal antibodies and Fc-fusion proteins. J Pharm Sci. 2015;104:1866-1884.

11. Baruah K, Bowden TA, Krishna BA, Dwek RA, Crispin M, Scanlan CN. Selective deactivation of serum IgG: a general strategy for the enhancement of monoclonal antibody receptor interactions. J Mol Biol. 2012;420:1-7.

12. Borrok MJ, Jung ST, Kang TH, Monzingo AF, Georgiou G. Revisiting the role of glycosylation in the structure of human IgG Fc. ACS Chem Biol. 2012;7:15961602.

13. Bruhns P, Iannascoli B, England $P$, et al. Specificity and affinity of human Fcgamma receptors and their polymorphic variants for human $\mathrm{IgG}$ subclasses. Blood. 2009;113:3716-3725.

14. Tuijnman WB, Van Wichen DF, Schuurman H-J. Tissue distribution of human IgG Fc receptors CD16, CD32 and CD64: an immunohistochemical study. APMIS. 1993;101:319-329.

15. Gao P, Pinkston KL, Wilganowski N, et al. Deglycosylation of mAb by EndoS for improved molecular imaging. Mol Imaging Biol. 2015;17:195-203.

16. Houghton JL, Zeglis BM, Abdel-Atti D, et al. Site-specifically labeled CA19.9targeted immunoconjugates for the PET, NIRF, and multimodal PET/NIRF imaging of pancreatic cancer. Proc Natl Acad Sci U S A. 2015;112:15850-15855.

17. Perk LR, Vosjan MJ, Visser GW, et al. p-Isothiocyanatobenzyl-desferrioxamine: a new bifunctional chelate for facile radiolabeling of monoclonal antibodies with zirconium-89 for immuno-PET imaging. Eur J Nucl Med Mol Imaging. 2010;37: 250-259.

18. Sharma SK, Chow A, Monette S, et al. Fc-mediated anomalous biodistribution of therapeutic antibodies in immunodeficient mouse models. Cancer Res. 2018;78: 1820-1832.

19. Cook BE, Adumeau P, Membreno R, et al. Pretargeted PET imaging using a sitespecifically labeled immunoconjugate. Bioconjugate Chem. 2016;27:1789-1795. 
20. Zeglis BM, Davis CB, Abdel-Atti D, et al. Chemoenzymatic strategy for the synthesis of site-specifically labeled immunoconjugates for multimodal PET and optical imaging. Bioconjugate Chem. 2014;25:2123-2128.

21. Zeglis BM, Lewis JS. The bioconjugation and eadiosynthesis of ${ }^{89} \mathrm{Zr}$-DFO-labeled antibodies. JoVE. 2015:e52521.

22. Adumeau P, Vivier D, Sharma SK, et al. Site-specifically labeled antibody-drug conjugate for simultaneous therapy and immunoPET. Mol Pharm. 2018;15:892898.

23. Li T, DiLillo DJ, Bournazos S, Giddens JP, Ravetch JV, Wang L-X. Modulating IgG effector function by Fc glycan engineering. Proc Natl Acad Sci U S A. 2017;114:3485-3490.

24. Shi Y, Fan X, Deng H, et al. Trastuzumab triggers phagocytic killing of high HER2 cancer cells in vitro and in vivo by interaction with $\mathrm{Fc} \gamma$ receptors on macrophages. J Immunol. 2015;194:4379-4386.

25. Deri MA, Zeglis BM, Francesconi LC, Lewis JS. PET imaging with ${ }^{89} \mathrm{Zr}$ : from radiochemistry to the clinic. Nucl Med Biol. 2013;40:3-14.

26. Collin M, Olsén A. EndoS, a novel secreted protein from Streptococcus pyogenes with endoglycosidase activity on human IgG. EMBO J. 2001;20:30463055 .

27. Zeglis BM, Davis CB, Aggeler R, et al. Enzyme-mediated methodology for the site-specific radiolabeling of antibodies based on catalyst-free click chemistry. Bioconjug Chem. 2013;24:1057-1067.

28. Dekkers G, Bentlage AEH, Stegmann TC, et al. Affinity of human IgG subclasses to mouse Fc gamma receptors. MAbs. 2017;9:767-773.

29. Overdijk MB, Verploegen S, Ortiz Buijsse A, et al. Crosstalk between human IgG isotypes and murine effector cells. J Immunol. 2012;189:3430-3438.

30. Bruhns P. Properties of mouse and human IgG receptors and their contribution to disease models. Blood. 2012;119:5640-5649.

31. Canfield SM, Morrison SL. The binding affinity of human IgG for its high affinity $\mathrm{Fc}$ receptor is determined by multiple amino acids in the $\mathrm{CH} 2$ domain and is modulated by the hinge region. J Exp Med. 1991;173:1483-1491.
32. Jayaram Y, Buckle AM, Hogg N. The Fc receptor, FcRI, and other activation molecules on human mononuclear phagocytes after treatment with interferongamma. Clin Exp Immunol. 1989;75:414-420.

33. Guilliams M, Bruhns P, Saeys Y, Hammad H, Lambrecht BN. The function of Fc $\gamma$ receptors in dendritic cells and macrophages. Nat Rev Immunol. 2014;14: 349.

34. Ishikawa F, Yasukawa M, Lyons B, et al. Development of functional human blood and immune systems in NOD/SCID/IL2 receptor $\gamma$ chain(null) mice. Blood. 2005; 106:1565-1573.

35. Baerenwaldt A, Lux A, Danzer H, et al. Fc $\gamma$ receptor IIB (Fc $\gamma$ RIIB) maintains humoral tolerance in the human immune system in vivo. Proc Natl Acad Sci USA. 2011;108:18772-18777.

36. Ito M, Shiina M, Saito Y, Tokuda Y, Kametani Y, Habu S. Antigen-specific antibody production of human B cells in NOG mice reconstituted with the human immune system. In: Nomura T, Watanabe T, Habu S, eds. Humanized Mice. Berlin, Germany: Springer Berlin Heidelberg; 2008:95-107.

37. Shultz LD, Ishikawa F, Greiner DL. Humanized mice in translational biomedical research. Nat Rev Immunol. 2007;7:118-130.

38. Brehm MA, Shultz LD, Luban J, Greiner DL. Overcoming current limitations in humanized mouse research. J Infect Dis. 2013;208(suppl 2):S125-S130.

39. Chen Q, He F, Kwang J, Chan JK, Chen J. GM-CSF and IL-4 stimulate antibody responses in humanized mice by promoting $\mathrm{T}, \mathrm{B}$, and dendritic cell maturation. J Immunol. 2012;189:5223-5229.

40. Abuqayyas L, Balthasar JP. Application of knockout mouse models to investigate the influence of FcgammaR on the tissue distribution and elimination of $8 \mathrm{C} 2$, a murine IgG1 monoclonal antibody. Int J Pharm. 2012;439:8-16.

41. Dijkers EC, Oude Munnink TH, Kosterink JG, et al. Biodistribution of ${ }^{89} \mathrm{Zr}$ trastuzumab and PET imaging of HER2-positive lesions in patients with metastatic breast cancer. Clin Pharmacol Ther. 2010;87:586-592.

42. Verma R, Foster RE, Horgan K, et al. Lymphocyte depletion and repopulation after chemotherapy for primary breast cancer. Breast Cancer Res. 2016;18:10. 